\title{
The effectiveness of prophylaxis for measles contacts in NSW
}

\section{Vicky Sheppeard ${ }^{\mathrm{A}, \mathrm{B}, \mathrm{F}}$, Bradley Forssman ${ }^{\mathrm{C}}$, Mark J. Ferson' ${ }^{\mathrm{D}}$, Conrad Moreira ${ }^{\mathrm{B}}$, Sue Campbell-Lloyd ${ }^{\mathrm{A}}$, Dominic E. Dwyer ${ }^{\mathrm{E}}$ and Jeremy M. McAnulty ${ }^{\mathrm{A}}$}

ACentre for Health Protection, NSW Department of Health

${ }^{\mathrm{B}}$ Centre for Population Health, Sydney West Area Health Service

${ }^{C}$ School of Public Health, University of Sydney

DPublic Health Unit, South Eastern Sydney Illawarra Area Health Service and School of Public Health and Community Medicine, University of NSW

${ }^{\mathrm{E}}$ Centre for Infectious Diseases and Microbiology Laboratory Services, Institute of Clinical Pathology and Medical Research, Westmead Hospital

${ }^{\mathrm{F} C o r r e s p o n d i n g}$ author. Email: vicky.sheppeard@ swahs.health.nsw.gov.au measles vaccine was first made available for infants. ${ }^{1} \mathrm{~A}$ second dose was introduced in New South Wales (NSW) for adolescent girls in 1992 and for boys in 1994. Since 1999, the vaccine has been recommended for children at 12 months and 4 or 5 years of age combined with mumps and rubella vaccine (MMR). In 1998, a school-based catch-up program provided a second MMR dose to primary school children. ${ }^{2}$ By 2005 , measles notifications in Australia had declined to the lowest ever recorded, and the NSW vaccination coverage rate was stable at around $94 \%$ for first dose and $89 \%$ for two doses. ${ }^{3}$

Measles is a notifiable condition in NSW, and public health follow-up involves interviews with the affected person or carer, advice about minimising spread to others, identification of exposed contacts, and provision of prophylactic immunisation, using either measles-containing vaccine (within 72 hours of exposure) or normal human immunoglobulin (NHIG) within 7 days of exposure. ${ }^{4}$ Measles may be infectious for many days before the diagnosis is made therefore many people may be exposed. Public health follow-up can be resource intensive but there are few data to indicate its effectiveness, and practice varies from country to country.

Recent analyses published by the United States (US) Centers for Disease Control and Prevention have confirmed the effectiveness of NHIG when given as post-exposure prophylaxis (PEP) at a dose of $0.25 \mathrm{~mL} / \mathrm{kg}$ up to 6 days after contact; however, one case occurred from those given MMR as prophylaxis., 5 A review in Japan raised some concerns regarding the efficacy of NHIG in children when used at a dose of $0.33 \mathrm{~mL} / \mathrm{kg}$ up to 5 days after exposure. ${ }^{7}$ We used the opportunity of measles outbreaks during March, April and May 2006 to assess the effectiveness of PEP. ${ }^{8}$

\section{Methods}

We obtained data on all notified cases of measles onset between 1 March and 31 May 2006 in NSW residents. We also included data on two residents of other states who acquired measles in NSW and required public health control activities by NSW public health units.

After a virtual absence of endemic measles transmission in Australia for 3 years, a large outbreak began in Sydney in March 2006. Measles vaccination rates in Australia have gradually increased since 1968, when single dose
Consistent with national recommendations, we defined a case as either laboratory-confirmed (either detection of measles-specific immunoglobulin $\mathrm{M}(\mathrm{IgM})$ antibodies in 
the presence of a compatible clinical illness, or detection of measles virus by immunofluorescence (IF) or reverse transcriptase-polymerase chain reaction (RT-PCR) or measles virus culture); or clinical (fever and/or cough and/or coryza and/or conjunctivitis and maculopapular rash) with an epidemiological link to a laboratory-confirmed case. ${ }^{4}$ All measles-specific IgM antibody tests were either performed initially or confirmed at a reference laboratory. Measles virus IF was performed on acetone-fixed smear swabs stained with measles-specific monoclonal antibodies and RT-PCR using primers to the measles nucleoprotein region on nasal or pharyngeal specimens, as previously described. ${ }^{9}$

Surveillance officers collected data on symptoms and signs of cases through interview of cases (or their parents) and their health-care providers, and recorded on a standard reporting form. Case interviews were also used to identify possible contacts.

A contact, as defined in the NSW Health protocol, was anyone who was in the same room as the case, or the same room for up to 2 hours after, during the infectious period. ${ }^{4}$ All contacts included in this study were Australian residents. Susceptible contacts, i.e. people who were considered to have inadequate immunity to measles, were defined according to Australian guidelines as:

- infants from 6-12 months of age;

- children aged 1-4 years who had not received any doses of MMR; and

- children aged over 4 years, and adults born during or after 1966, who had not received two doses of MMR. ${ }^{10}$

Contacts identified as susceptible were offered postexposure prophylactic immunisation with either MMR within 3 days of exposure, or NHIG within 7 days of exposure.

Other public health actions included:

- advising contacts about the symptoms of measles and how to avoid infecting other people;

- utilising mass media messages;

- enhanced surveillance using direct communication via faxes to general practitioners, hospitals, child-care centres and laboratories to raise awareness of the outbreak; and

- extension of eligibility for free MMR vaccine from general practitioners to all susceptible persons in NSW from 18 May 2006.

Data on cases, contacts and prophylaxis were collated using Microsoft Excel 2000 (Microsoft Corporation).
Prophylaxis effectiveness (PE) was calculated using the cohort method:

$$
\mathrm{PE}=((\mathrm{ARU}-\mathrm{ARP}) / \mathrm{ARU}) \times 100)
$$

where ARU represents the attack rate in susceptible contacts not receiving prophylaxis and ARP is the attack rate in susceptible contacts who received prophylaxis. ${ }^{11}$ Taylor series 95\% confidence intervals were calculated around the relative risk, and then converted to PE confidence intervals. $^{12}$

Rates of secondary cases in contact groups were compared using Fisher's exact test in EpiInfo 3.5.1 (Centers for Disease Control and Prevention).

\section{Results}

\section{Description of the outbreak}

Fifty-seven cases of measles were reported during the 3-month period (Figure 1). A description of the first 2 months of the outbreak has been reported elsewhere. ${ }^{8,13}$

Of the 57 cases, four (7\%) were aged under 1 year, 19 (33\%) 1-4 years, 14 (25\%) 5-14 years, 18 (32\%) 15-40 years and $2(4 \%)$ over 40 years. Thirteen $(23 \%)$ required hospitalisation. Hospital admission was more common in infants and adults (38\%) than in children aged $1-14$ years $(12 \%)$.

\section{Public health interventions}

In total, 1760 contacts were identified, an average of 31 contacts per case. Five hundred and fifty-three contacts (31\%) were defined as susceptible to measles. Twelve patients spent time in busy hospital emergency departments while infectious and prior to being diagnosed, resulting in 1139 of these identified contacts.

Early in the outbreak, delays in clinical and laboratory confirmation of measles and case notification as well as delays in identification of potential contacts, meant that prophylactic immunisation for contacts within the recommended 7 days of exposure was often not possible. ${ }^{4}$ For example, an early case visited the hospital emergency department four times prior to diagnosis, and 120 families who were exposed were not identified within 7 days.

Table 1 summarises prophylactic administration for susceptible contacts and numbers of secondary cases according to prophylaxis type. Other contacts managed by their general practitioner or hospital are not included.

Of the 265 contacts known to have received prophylaxis, only two $(0.8 \%)$ subsequently developed measles. Both of these contacts received NHIG on the seventh day following contact with a case. Of the 288 susceptible contacts who did not receive any prophylaxis, 13 (4.5\%) developed 


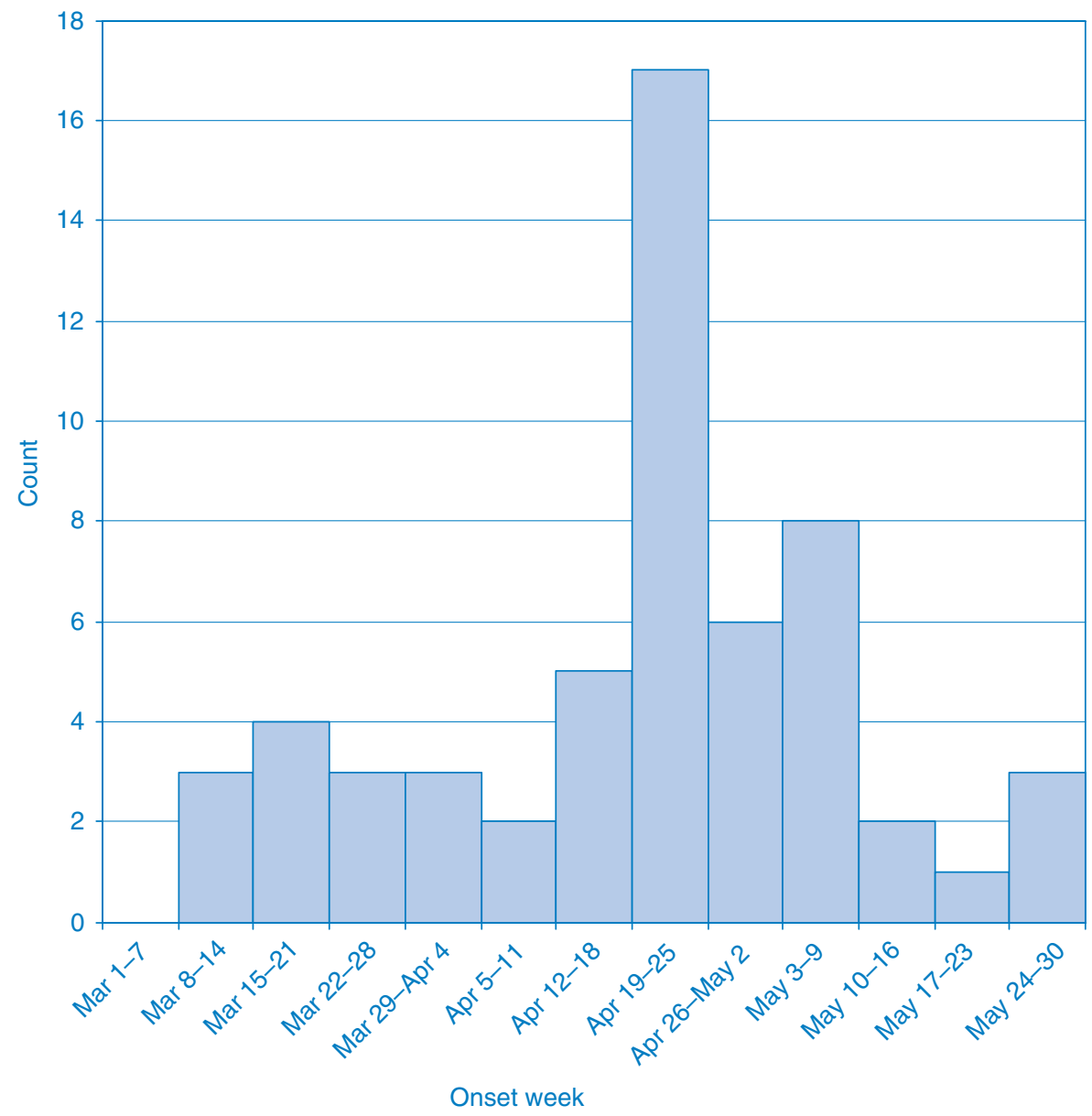

Figure 1. Notified cases by week of onset, NSW, March-May 2006.

Table 1. Prophylaxis and outcomes for susceptible contacts of measles cases, NSW, March-May 2006

\begin{tabular}{lccc}
\hline & $\begin{array}{c}\text { Contacts } \\
\text { identified }\end{array}$ & $\begin{array}{c}\text { Secondary cases } \\
\text { identified }\end{array}$ & $\begin{array}{c}\text { Rate } \\
\text { (per 1000) }\end{array}$ \\
\hline MMR & 82 & 0 & 0 \\
NHIG & 183 & 2 & 10.9 \\
Refused & 93 & 3 & 32.3 \\
Too late & 195 & 10 & 51.3 \\
Total & 553 & 15 & 27.1 \\
\hline \multicolumn{4}{l}{ MMR: measles-mumps-rubella vaccine. } \\
NHIG: normal human immunoglobulin.
\end{tabular}

measles. Applying this percentage to the 265 contacts known to have received prophylaxis, 12 (rather than two) may have otherwise developed measles.

The effectiveness of receiving either MMR or NHIG as prophylaxis compared to no prophylaxis in susceptible contacts was $83.3 \%$ (95\% CI: 27-96). The effectiveness of prophylaxis with NHIG alone was 75.8\% (CI: 0-94) and for MMR was $100 \%$.
The rate of secondary measles was significantly higher among social contacts (eight cases from 117 identified contacts) and household contacts (five from 128) compared to either of the two cases that arose from 1139 identified hospital contacts, and none from 400 school or child-care contacts $(P<0.0002)$.

\section{Discussion}

In the largest outbreak of measles in NSW since the National Measles Control Campaign, public health interventions including post-exposure prophylaxis were effective in preventing the further spread of measles. Although perhaps only 10 secondary cases were directly averted, those additional 10 cases could have generated further generations of cases and their contacts.

This study had a number of limitations. First, contacts' susceptibility was estimated based on Australian guidelines and were not serologically confirmed. Classification on the basis of age could lead to either an over- or underestimation of the number of susceptible contacts. Our data on the rate of measles in contacts who did not receive prophylaxis suggest that perhaps as few as $4.5 \%$ of contacts 
who we defined as susceptible were in fact susceptible, and so the number of contacts categorised as susceptible was likely an overestimate. Any misclassification was, however, non-differential between those who did and did not receive prophylaxis, which would result in an underestimate of effectiveness. Second, the estimation of effectiveness may be affected by under enumeration of secondary cases. It is possible that secondary cases of measles were not detected if they did not seek health care or were misdiagnosed. However, we believe this is unlikely due to the assertive public health actions. Finally, we did not record data on all contacts as some contact tracing efforts were managed outside of public health units.

Despite these limitations, this study has demonstrated that PEP was effective in reducing this measles outbreak, and is likely to be so in the future. Such outbreaks have been predicted because a proportion of the population remains susceptible to measles. ${ }^{14,15}$ Each year, 5-10\% of eligible Australian children are not vaccinated, there is an expected vaccine failure rate and a known susceptibility in young adults born after 1965.16 The success of Australia's measles vaccination programs has largely resulted in the elimination of local transmission of measles in the past decade. ${ }^{3}$ Thus, health-care providers and the community are less familiar with its presentation, severity and potential for complications. This presents a challenge in maintaining a high index of suspicion for measles diagnosis (including appropriate laboratory confirmation) and ensuring an appropriate outbreak response from both health-care providers and the community. Therefore, a timely response by public health agencies to every measles notification is critical to effective measles control.

We estimated that the effectiveness of prophylaxis with either NHIG or MMR was $83 \%$, and that both types of prophylaxis were effective. When MMR was given within 3 days of exposure, no cases of measles occurred. Measles did arise in two recipients of NHIG but both of these contacts received prophylaxis on the seventh day after exposure. This suggests that the effectiveness of NHIG prophylaxis decreases with time since a contact is exposed to measles, and may not be useful more than 6 days after exposure.

There are only a small number of recent reports on the value of prophylaxis during measles outbreaks. In an outbreak in Iowa, United States, no cases of measles arose in 20 contacts given NHIG prophylaxis within 6 days of contact with measles; however, one case arose among 175 persons receiving post-exposure MMR. ${ }^{5}$ In an outbreak in Japan, NHIG appeared to be less effective as $57 \%$ of contacts given NHIG developed measles. ${ }^{7}$

The apparently greater effectiveness of prophylaxis in the Iowa and NSW outbreaks may be due to the dose of NHIG used for measles prevention: the recommended NHIG dose for measles prophylaxis in Australia is $0.2 \mathrm{~mL} / \mathrm{kg}$ and $0.25 \mathrm{~mL} / \mathrm{kg}$ in the US, whereas in Japan $0.33 \mathrm{~mL} / \mathrm{kg}$ of NHIG is used. ${ }^{4,7,17}$ However, the reported titre of measlesspecific IgG in the Japanese NHIG was equal to or less than $16 \mathrm{IU} / \mathrm{mL}$, while the titre of measles-specific $\mathrm{IgG}$ in the Australian NHIG preparation is currently estimated to be $32 \mathrm{IU} / \mathrm{mL}$ (personal communication, Trish Kleeman, CSL Limited, 19 April 2007). This suggests that if a high level of immunity is maintained in the community then adequate measles antibody titres will be present in NHIG preparations derived from blood donations.

\section{Conclusion}

Measles outbreaks create a substantial burden on the public health system. In the recent outbreak in Iowa, the economic impact of containing one case of measles was estimated at \$US142 452, largely in time spent by public health staff on contact tracing and giving prophylaxis. ${ }^{6}$

In our study, 176 contacts were traced and 26 people given post-exposure prophylaxis for each case of measles averted. Although only 10 secondary cases of measles may have been averted, many subsequent generations of disease and the follow-up of scores of associated contacts were also likely to have been prevented.

Post-exposure immunisation remains an effective tool for preventing secondary cases of measles.

\section{Acknowledgments}

The NSW Public Health Network, particularly communicable disease surveillance officers, the Virology Section of the Institute for Clinical Pathology and Medical Research and NSW Department of Health Communicable Diseases Branch and Immunisation Unit all contributed extensively. Alex Rosewell assisted with the review of emergency department records to identify the index patient.

\section{References}

1. Gidding HF, Burgess MA, Kempe AE. A short history of vaccination in Australia. Med J Aust 2001; 174: 37-40.

2. Turnbull FM, Burgess MA, McIntyre PB, Lambert SB, Gilbert GL, Gidding HF et al. The Australian Measles Control Campaign, 1998. Bull World Health Organ 2001; 79: 882-8.

3. Brotherton J, Wang H, Schaffer A, Quinn H, Menzies R, Hull B et al. Vaccine preventable diseases and vaccination coverage in Australia 2003 to 2005. Commun Dis Intell 2007; 31(Suppl): S31-6.

4. NSW Department of Health. Measles response protocol for public health units. Sydney: NSW Department of Health; 2004. Available from http://www.health.nsw.gov.au/infect/ pdf/measles.pdf (Cited 20 March 2006.)

5. Centers for Disease Control and Prevention. Postexposure prophylaxis, isolation, and quarantine to control an importassociated measles outbreak - Iowa, 2004. MMWR Morb Mortal Wkly Rep 2004; 53: 969-71.

6. Dayan GH, Ortega-Sanchez IR, LeBaron CW, Quinlisk MP. The cost of containing one case of measles: The Economic 
Impact on the Public Health Infrastructure - Iowa, 2004. Pediatrics 2005; 116: e1-4. doi:10.1542/peds.2004-2512

7. Endo A, Izumi H, Miyashita M, Taniguchi K, Okubo O, Harada K. Current efficacy of postexposure prophylaxis against measles with immunoglobulin. J Pediatr 2001; 138: 926-8. doi:10.1067/mpd.2001.113710

8. Department of Health and Aging. Communicable Diseases Surveillance - Highlights for 2nd quarter, 2006. CDI 2006; 30(3): 385-7. Available from: http://www.health.gov.au/ internet/main/publishing.nsf/Content/cda-cdi3003n.htm (Cited November 2008.)

9. Weston KM, Dwyer DE, Ratnamohan M, McPhie K, Chan SW, Branley JM et al. Nosocomial and community transmission of measles virus genotype D8 by a returning traveller from Nepal. Commun Dis Intell 2006; 30: 58-65.

10. Communicable Diseases Network Australia and New Zealand. Guidelines for the control of measles outbreaks in Australia. Technical Report Series No. 5. Canberra: Commonwealth Department of Health and Aged Care; 2000.

11. Torvaldsen S, McIntyre PB. Observational methods in epidemiologic assessment of vaccine effectiveness. Commun Dis Intell 2002; 26: 451-7.
12. Hightower AW, Orenstein WA, Martin SM. Recommendations for the use of Taylor series confidence intervals for estimates of vaccine efficacy. Bull World Health Organ 1988; 66: 99-105.

13. Communicable Diseases Report. New South Wales, for March and April 2006. NSW Public Health Bull 2006; 17: 88-94. doi:10.1071/NB06022

14. MacIntyre CR, Hull B, Burgess M. Measles control in NSW Divisions of General Practice. NSW Public Health Bull 2003; 14: 13-6. doi:10.1071/NB03004

15. Parker AA, Staggs W, Dayan GH, Ortega-Sánchez IR, Rota PA, Lowe L et al. Implications of a 2005 measles outbreak in Indiana for sustained elimination of measles in the United States. N Engl J Med 2006; 355: 447-55. doi:10.1056/NEJMoa060775

16. Gidding HF, Gilbert GL. Measles immunity in young Australian adults. Commun Dis Intell 2001; 25: 133-6.

17. Heyman DL, editor. Control of communicable diseases manual. 18th ed. Washington: American Public Health Association; 2004. 